\title{
Primal worst and dual best in robust vector optimization
}

\author{
Elisa Caprari $^{1}$, Lorenzo Cerboni Baiardi $^{1}$, Elena Molho ${ }^{1}$ \\ ${ }^{1}$ Department of Economics and Management, University of Pavia (Italy)
}

\begin{abstract}
We establish a relationship between the robust counterpart of an uncertain cone-convex vector problem and the optimistic counterpart of its uncertain dual. Along the line marked by Beck and Ben-Tal (2009) in the scalar case, we show that operating in the primal problem with a pessimistic view is equivalent to operating with an optimistic approach in its dual.

Keywords: multiple objective programming; vector optimization problems; Lagrangian duality; robust optimization.
\end{abstract}

\section{Introduction}

We consider vector optimization problems where the objective and the constraint depend on uncertain parameters. The robust approach (introduced in [4]) hedges the decision maker against the worst cases that may occur as the uncertainties vary within their domains and it is suitable to tackle the situation where no probability distribution on uncertain parameters is available. In this paper we study the relationship between the robust counterpart of the uncertain primal problem and its uncertain dual problem. The relation involves the notion of optimistic counterpart where, in contrast with the robust approach, the decision maker faces the indeterminacy by assuming that the occurrence of the uncertain parameters are the most favorable ones. This is along the line marked by [2], where the authors show that, for an uncertain scalar convex program, operating with a pessimistic view in the primal is the same as operating with an optimistic view in the dual ("primal worst equals dual best"). However, in the framework of uncertain vector optimization problems, the values of the uncertain objective at each choice of the decision variable individuate a set and the optimization of worst (and best) scenarios have an intrinsic set-valued nature. Following a set optimization approach, we consider optimality with respect to the upper quasiorder relation $\prec_{K}$ among sets. Since the primal is a minimization problem, this choice leads to a robust optimization approach, where only worst scenarios are considered. On the other hand, the use of the upper quasiorder relation $\prec_{K}$ in the dual maximin problem, implies that only best scenarios are considered, hence an optimistic approach is intrinsically adopted.

For our results to be valid we require the cone-convexity of the set-valued objective and constraint involved in the robust counterpart. Moreover, the analysis here 
considered is limited to those uncertain problems where, at each given value of the decision variable, a single worst case exists that can be compared with all the other scenarios. Despite this restriction, the result can be applied to a wide variety of practical instances such as, for example, the case of interval-wise uncertainties, that includes multiobjective linear programs with interval coefficients.

The paper is organized as follows. In Section 3 we provide an extension to set optimization of classical strong duality results for scalar programs (see [1], among others). In Section 4 we define the robust counterpart of an uncertain vector problem, the optimistic counterpart of its uncertain dual and, in Section 5, we compare, if possible, their solutions in the image set. In Section 6 we show that our results can be applied for instance to those vector problems with interval-wise uncertainties, with application to a multiobjective supplier selection model. Section 7 concludes.

\section{Preliminary notions}

Let $Y$ be a normed linear space and let $K \subset Y$ be a cone assumed closed, pointed $(K \cap\{-K\}=\{0\})$ convex and solid (that is $\operatorname{int} K \neq \emptyset$ where $\operatorname{int} K$ denotes the topological interior of $K$ ). Let $Y^{*}$ be the topological dual space of $Y$ and let $K^{+}$be the dual cone defined as

$$
K^{+}:=\left\{\varphi \in Y^{*}: \varphi(k) \geqslant 0, \forall k \in K\right\}
$$

In the present work, the following order structure induced on $Y$ by the cone $K$ is of interest.

Definition 2.1. Let $y_{1}, y_{2} \in Y$. Then

$$
\begin{aligned}
& y_{1} \leq_{K} y_{2} \Longleftrightarrow y_{2}-y_{1} \in K \\
& y_{1}<_{K} y_{2} \Longleftrightarrow y_{2}-y_{1} \in \operatorname{int} K
\end{aligned}
$$

Relations defined in 2.1 allow to define minimal/maximal (resp. weakly mini$\mathrm{mal} /$ maximal) elements of a set w.r.t. the ordering cone.

Definition 2.2. Let $A \subset Y$ be a nonempty set and let $K$ be a pointed, solid, closed and convex ordering cone. The element $y \in A$ is said to be

i) a minimal (resp. weakly minimal) element of $A$ w.r.t. the ordering cone $K$ if

$$
A \cap(y-K)=\{y\} \quad(\text { resp. } A \cap(y-\operatorname{int} K)=\emptyset)
$$

The set of minimal (resp. weakly minimal) elements of the set $A$ w.r.t. $K$ are denoted by $\operatorname{Min}_{K} A$ (resp. $\mathrm{WMin}_{K} A$ );

ii) a maximal (resp. weakly maximal) element of $A$ w.r.t. the ordering cone $K$ if

$$
A \cap(y+K)=\{y\} \quad(\operatorname{resp} . A \cap(y+\operatorname{int} K)=\emptyset)
$$

The set of maximal (resp. weakly maximal) elements of the set $A$ w.r.t. $K$ are denoted by $\operatorname{Max}_{K} A$ (resp. $\left.\mathrm{WMax}_{K} A\right)$.

Remark 2.3. The above definitions imply that $y \in A$ is a minimal (resp. weakly minimal) element of $A$ if no element $y^{\prime} \in A$ with $y^{\prime} \neq y$ exists such that $y^{\prime} \in y-K$ (resp. $y^{\prime} \in y-\operatorname{int} K$ ). Analogous considerations can be made for maximal (resp. weakly maximal) elements of a set $A$. 
Let us denote by $2^{Y}$ the power set of $Y$, namely the collection of all nonempty subsets of $Y$. In the present work, the following relations induced onto $2^{Y}$ by the ordering cone $K$ are of interest. We choose the so called upper type quasiorder relation (see [16] and [17]), since it is the most suitable to be applied to robust optimization.

Definition 2.4. Let $A, B \in 2^{Y}$. Then

$$
\begin{aligned}
& A \prec_{K} B \quad \Longleftrightarrow A \subseteq B-K \\
& A \prec_{K} B \Longleftrightarrow A \subseteq B-\operatorname{int} K
\end{aligned}
$$

Definition 2.5. Let $A, B \in 2^{Y}$. The equivalence relation between $A$ and $B$ w.r.t. the order (3a) is defined as follows (see [17]):

$$
A \sim_{K} B \Longleftrightarrow A \prec_{K} B \text { and } B \prec_{K} A
$$

Equivalence classes w.r.t. the order $\prec_{K}$ are denoted by $[\cdot]_{K}$, meaning that if $B \in[A]_{K}$ then $A \prec_{K} B$ and $B \prec_{K} A$.

For the proof of the following lemma see [10].

Lemma 2.6. Let $A, B \in \mathcal{A}$. If $A \prec_{K} B$ and $B \prec_{K} A$ then $A \sim_{K} B$

The following set-relation will also be considered.

Definition 2.7. Let $A, B \in \mathcal{A}$. Then

$$
A \nprec_{K} B \Longleftrightarrow A \nsubseteq B-\operatorname{int} K
$$

Definition 2.8. Let $A, B \in 2^{Y}$. $A$ and $B$ are said to be non comparable w.r.t. the order $\prec_{K}$ if $A \nprec_{K} B$ and $B \nprec_{K} A$ and not $A \sim_{K} B$.

The following notions of minimality of elements of a collection of sets $\mathcal{A} \subset 2^{Y}$ with respect to $K$ are based on the set-relations defined in 2.4 .

Definition 2.9. Let $\mathcal{A} \subset 2^{Y}$ be a collection of sets. A nonempty set $A \in \mathcal{A}$ is said:

i) a $\prec_{K}-$ minimal (resp. $\prec_{K}-$ minimal) set of $\mathcal{A}$ if

$$
B \in \mathcal{A} \text { and } B \prec_{K} A\left(\text { resp. } B \prec_{K} A\right) \Longrightarrow A \prec_{K} B\left(\text { resp. } A \prec_{K} B\right)
$$

The family of $\prec_{K}-$ minimal (resp. $\prec_{K}-$ minimal) sets of $\mathcal{A}$ will be denoted as $\prec_{\mathrm{K}}-\min \mathcal{A}\left(\right.$ resp. $\left.\prec_{\mathrm{K}}-\min \mathcal{A}\right)$.

ii) a $\prec_{\mathrm{K}}-$ maximal (resp. $\prec_{K}-$ maximal) set of $\mathcal{A}$ if

$$
B \in \mathcal{A} \text { and } A \prec_{K} B\left(\text { resp. } A \prec_{K} B\right) \Longrightarrow B \prec_{K} A\left(\text { resp. } B \prec_{K} A\right)
$$

The family of $\prec_{K}$-maximal (resp. $\prec_{K}$-maximal) sets of $\mathcal{A}$ will be denoted as $\prec_{\mathrm{K}}-\max \mathcal{A}\left(\right.$ resp. $\left.\prec_{\mathrm{K}}-\max \mathcal{A}\right)$.

From now on, let $X, Y$ and $Z$ be real normed linear spaces. The image spaces $Y$ and $Z$ are endowed with an order structure induced respectively by the closed, convex, pointed and solid cones $K$ and $C$ respectively. 
Definition 2.10. A set-valued map $F: X \rightrightarrows Y$ is said to be $\prec_{K}$-convex on the convex set $A \subseteq X$ when

$$
F\left(\alpha x_{1}+(1-\alpha) x_{2}\right) \prec_{K} \alpha F\left(x_{1}\right)+(1-\alpha) F\left(x_{2}\right)
$$

for every $\alpha \in[0,1]$ and for every $x_{1}, x_{2} \in A$.

Definition 2.11. The upper epigraph of $F(x)$ is the set $\left\{\prec_{\mathrm{K}}-\operatorname{epi} F\right\} \subseteq X \times Y$ defined as $\left\{\prec_{\mathrm{K}}-\mathrm{epi} F\right\}:=\left\{(x, y) \in X \times Y: F(x) \prec_{K}\{y\}\right\}$

Proposition 2.12. If the set-valued map $F: X \rightrightarrows Y$ is $\prec_{K^{-}}$-convex on $X$, then $\left\{\prec_{\mathrm{K}}-\mathrm{epi} F\right\}$ is a convex set.

Proof. Let $\left(x_{1}, y_{1}\right)$ and $\left(x_{2}, y_{2}\right)$ be elements of $\left\{\prec_{\mathrm{K}}-\mathrm{epi} F\right\}$. Hence

$$
F\left(x_{1}\right) \subseteq y_{1}-K \text { and } F\left(x_{2}\right) \subseteq y_{2}-K
$$

It follows that, $\forall \alpha \in[0,1]$, it holds:

$$
\alpha F\left(x_{1}\right)+(1-\alpha) F\left(x_{2}\right) \subseteq \alpha y_{1}+(1-\alpha) y_{2}-K
$$

therefore

$$
F\left(\alpha x_{1}+(1-\alpha) x_{2}\right) \subseteq \alpha F\left(x_{1}\right)+(1-\alpha) F\left(x_{2}\right)-K \subseteq \alpha y_{1}+(1-\alpha) y_{2}-K .
$$

thus showing that $\alpha\left(x_{1}, y_{1}\right)+(1-\alpha)\left(x_{2}, y_{2}\right) \in\left\{\prec_{\mathrm{K}}-\mathrm{epi} F\right\}$.

Definition 2.13. Let $A \subset Y$ be a set. The element $\mu(A) \in Y$ is said to be a nadir point of $A$ if $\mu(A) \in A$ and $A \prec_{K}\{\mu(A)\}$.

The following properties are easily proved.

Lemma 2.14. Let $A, B \subset Y$ be nonempty sets and assume the existence of their nadir points $\mu(A)$ and $\mu(B)$, respectively.

i) $A-K=\mu(A)-K$;

ii) $A-\operatorname{int} K=\mu(A)-\operatorname{int} K$;

iii) $A \prec_{K} B \Longleftrightarrow \mu(A) \leq_{K} \mu(B)$;

iv) $A \prec_{K} B \Longleftrightarrow \mu(A)<_{K} \mu(B)$;

v) $\mu(A+B)$ exists and $\mu(A+B)=\mu(A)+\mu(B)$.

\section{Some duality results in set optimization}

Let $Y$ and $Z$ be normed linear spaces and let $K \subset Y$ and $C \subset Z$ be closed, pointed convex and solid ordering cones. Let us consider the set-valued maps $F: X \rightrightarrows Y$ and $G: X \rightrightarrows Z$ and the following set-valued optimization problem

$$
\prec_{\mathrm{K}}-\min F(x) \text { subject to } G(x) \prec_{C}\{0\}
$$

Definition 3.1. An element $x \in X$ is said to be feasible for $(P)$ if $G(x) \prec_{C}\{0\}$.

Definition 3.2. A feasible element $x_{0} \in X$ for the problem $(P)$ is said to be a solution of $(P)$ if the existence of $x^{\prime} \in X$ such that $G\left(x^{\prime}\right) \prec_{C}\{0\}$ and $F\left(x^{\prime}\right) \prec_{K} F\left(x_{0}\right)$ implies $F\left(x_{0}\right) \prec_{K} F\left(x^{\prime}\right)$. 
Following the approach introduced in [16] (and considered in [10] and [9]), we formulate the set-valued dual problem of the primal $(P)$ by adapting the involved definitions, originally referred to a different order relation among sets, to the upper quasiorder relation $\prec_{K}$. Let $\mathcal{L}^{+}(Z, Y)$ be the set of monotone continuous linear operators from $Z$ to $Y$, i.e. $\Lambda \in \mathcal{L}^{+}(Z, Y)$ when $\Lambda(C) \subseteq K$. The Lagrangian function associated to $(P)$ is the set-valued map $L: X \times \mathcal{L}^{+}(Z, Y) \rightrightarrows Y$ defined by

$$
L(x, \Lambda)=F(x)+(\Lambda \circ G)(x)
$$

where $(\Lambda \circ G)(x)=\bigcup_{z \in G(x)} \Lambda(z)$. Let

$$
\Phi(\Lambda)=\prec_{\mathrm{K}}-\min \{L(x, \Lambda): x \in X\}
$$

At any $\Lambda$, the objective $\Phi(\Lambda)$ is a collection of sets that includes all $L(x, \Lambda)$ that are minimal w.r.t. the quasiorder relation $\prec_{K}$ as the variable $x$ varies in $X$. In terms of the objective $\Phi(\Lambda)$, the dual problem of $(P)$ is formulated as

$$
\prec_{K}-\operatorname{MAX} \Phi(\Lambda) \quad \text { s.t. } \Lambda \in \mathcal{L}^{+}(Z, Y)
$$

Due to the fact that $\Phi$ is a collection of sets at any given $\Lambda$, we need to define a solution concept for $(D)$.

Definition 3.3. The pair $(x, \Lambda)$ is feasible for $(D)$ if $L(x, \Lambda) \in \Phi(\Lambda)$ and $\Lambda \in$ $\mathcal{L}^{+}(Z, Y)$.

Definition 3.4. An element $\Lambda_{1} \in \mathcal{L}^{+}(Z, Y)$ is said to be a solution of $(D)$ if there exists an element $x_{1} \in X$ such that $L\left(x_{1}, \Lambda_{1}\right) \in \Phi\left(\Lambda_{1}\right)$ and

$$
L\left(x_{1}, \Lambda_{1}\right) \nprec_{K} L\left(x^{\prime}, \Lambda^{\prime}\right)
$$

for all feasible pairs $\left(x^{\prime}, \Lambda^{\prime}\right)$ of $(D)$.

We provide a weak duality Theorem by adapting the results originally obtained in [16] for a different quasiorder relation among sets (see also [10] and [9]) to the upper quasiorder structure $\prec_{K}$ considered here.

Theorem 3.5 (Weak Duality). Let $x_{0}$ be a feasible element of $(P)$ and let $\left(x_{1}, \Lambda_{1}\right)$ be a feasible pair of $(D)$. If $F\left(x_{0}\right) \prec_{K} L\left(x_{1}, \Lambda_{1}\right)$, then $F\left(x_{0}\right) \sim_{K} L\left(x_{1}, \Lambda_{1}\right)$.

Proof. Let $F\left(x_{0}\right) \prec_{K} L\left(x_{1}, \Lambda_{1}\right)$. Since $\left(\Lambda_{1} \circ G\right)\left(x_{0}\right) \subseteq-K$, being $G\left(x_{0}\right) \prec_{C}\{0\}$ and $\Lambda_{1} \in \mathcal{L}^{+}(Z, Y)$, it holds:

$$
L\left(x_{0}, \Lambda_{1}\right) \prec_{K} F\left(x_{0}\right)
$$

hence

$$
L\left(x_{0}, \Lambda_{1}\right) \prec_{K} L\left(x_{1}, \Lambda_{1}\right)
$$

In addition, since $L\left(x_{1}, \Lambda_{1}\right) \in \Phi\left(\Lambda_{1}\right)$, it is also

$$
L\left(x_{1}, \Lambda_{1}\right) \prec_{K} L\left(x_{0}, \Lambda_{1}\right)
$$

Relations (12) and (10) lead to

$$
L\left(x_{1}, \Lambda_{1}\right) \prec_{K} F\left(x_{0}\right)
$$

and, from Lemma 2.6, the thesis follows.

We will consider the following assumption. 
Assumption 3.6. Let $F: X \rightrightarrows Y$ and $G: X \rightrightarrows Z$. The nadir points $\mu(F(x))$ and $\mu(G(x))$ of $F(x)$ and $G(x)$ respectively exist for all $x \in X$.

Remark 3.7. If we consider the special case of a vector optimization problem, where the image sets at any value of the decision variable shrinks to a singleton, Assumption 3.6 holds.

Under Assumption 3.6, the weak duality result of Theorem 3.5 can be reformulated as follows.

Corollary 3.8. Let $x_{0}$ be a feasible element of $(P)$ and let $\left(x_{1}, \Lambda_{1}\right)$ be a feasible pair of $(D)$. Then, under Assumption 3.6, it holds

$$
F\left(x_{0}\right) \nprec_{K} L\left(x_{1}, \Lambda_{1}\right)
$$

In the sequel of this section we prove a strong duality result. Since, in set optimization, a partial quasiorder is used to compare images of objective maps, the duality gap does not allow us to obtain an equality between optimal values of the primal and of the dual problems; we can only establish either their equivalence or their non comparability.

In order to provide a strong duality result, the following Lemmas will be useful.

Lemma 3.9. Let $F: X \rightrightarrows Y$ and $G: X \rightrightarrows Z$ be $\prec_{K^{-}}$-convex and $\prec_{C^{-}}$-convex maps respectively and let

$$
Q(x)=\left\{y \in Y: F(x) \prec_{K}\{y\}\right\} \times\left\{z \in Z: G(x) \prec_{C}\{z\}\right\}
$$

Then the set

$$
Q(X)=\bigcup_{x \in X} Q(x)
$$

is convex.

Proof. Since $F$ is $\prec_{K}$-convex the set $\left\{\prec_{K}-\mathrm{epi} F\right\}$ is convex (see Proposition 2.12), for all $\left(x_{1}, y_{1}\right),\left(x_{2}, y_{2}\right) \in\left\{\prec_{K}-\mathrm{epi} F\right\}$ and for all $\alpha \in[0,1]$

$$
\alpha\left(x_{1}, y_{1}\right)+(1-\alpha)\left(x_{2}, y_{2}\right)=\left(\alpha x_{1}+(1-\alpha) x_{2}, \alpha y_{1}+(1-\alpha) y_{2}\right) \in\{\prec-\mathrm{epi} F\}
$$

that is

$$
F\left(\alpha x_{1}+(1-\alpha) x_{2}\right) \prec_{K}\left\{\alpha y_{1}+(1-\alpha) y_{2}\right\}
$$

The same argument shows that the set $\left\{\prec_{C}-\operatorname{epi} G\right\}$ is also convex since, by hypotheses, $G$ is $\prec_{C}$-convex. Consider now $\left(y_{1}, z_{1}\right) \in Q\left(x_{1}\right),\left(y_{2}, z_{2}\right) \in Q\left(x_{2}\right)$ and $\alpha \in[0,1]$. Since $\left(x_{1}, y_{1}\right),\left(x_{2}, y_{2}\right) \in\left\{\prec_{K}-\mathrm{epi} F\right\}$ and $\left(x_{1}, z_{1}\right),\left(x_{2}, z_{2}\right) \in\left\{\prec_{C}-\right.$ epi $\left.G\right\}$ and they are convex, we have

$\alpha\left(y_{1}, z_{1}\right)+(1-\alpha)\left(y_{2}, z_{2}\right)=\left(\alpha y_{1}+(1-\alpha) y_{2}, \alpha z_{1}+(1-\alpha) z_{2}\right) \in Q\left(\alpha x_{1}+(1-\alpha) x_{2}\right)$

Hence $Q(X)$ is convex.

Lemma 3.10. Let $x_{0}$ be a solution of $(P)$ and let

$$
A(y)=(y-K) \times(-C)
$$

Under Assumption 3.6, it holds

$$
Q(X) \bigcap \operatorname{int} A\left(y_{0}\right)=\emptyset
$$

where $y_{0}=\mu\left(F\left(x_{0}\right)\right)$. 
Proof. By contradiction, let $\left(y_{1}, z_{1}\right) \in Q\left(x_{1}\right)$ be such that $y_{1} \in y_{0}-\operatorname{int} K$ and $z_{1} \in$ $-\operatorname{int} C$. It holds $F\left(x_{1}\right) \subseteq y_{1}-K \subseteq y_{0}-\operatorname{int} K$, hence $F\left(x_{1}\right) \prec_{K} F\left(x_{0}\right)$ and $G\left(x_{1}\right) \prec_{C}$ $\{0\}$. Since $x_{0}$ is a solution of $(P)$, if $G\left(x_{1}\right) \prec_{C}\{0\}$, then $F\left(x_{1}\right) \sim_{K} F\left(x_{0}\right)$. Therefore

$$
y_{0}-K=F\left(x_{0}\right)-K=F\left(x_{1}\right)-K
$$

This, together with $F\left(x_{1}\right) \prec_{K}\left\{y_{1}\right\}$, leads to $y_{0} \in F\left(x_{1}\right)-K \subseteq y_{1}-K$. Hence $y_{1}-y_{0} \in K \cap-\operatorname{int} K$, a contradiction, since $K$ is pointed.

For the convenience of the reader, we provide the separation result given in the first part of Theorem 6.2.5 in [15].

Theorem 3.11. Let $W$ be a separated locally convex space and let $A, B \subset W$ be nonempty convex sets. Then, if $\operatorname{int} A \neq \emptyset$ and $B \cup \operatorname{int} A=\emptyset$, or more generally, if $0 \in \operatorname{int}(A-B) \neq \emptyset$, then there exists $w^{*} \in W^{*} \backslash\{0\}$ such that $\sup w^{*}(A) \leq \inf w^{*}(B)$.

In the sequel, we say that the Slater Constraint Qualification (SCQ) holds when an element $\hat{x} \in X$ exists such that $G(\hat{x}) \prec_{C}\{0\}$.

Theorem 3.12 (Strong Duality). Let $F: X \rightrightarrows Y$ and $G: X \rightrightarrows Z$ be $\prec_{K^{-}}$-convex and $\prec_{C}$-convex maps respectively on the convex set $X$ and the SCQ holds. Under Assumption 3.6, if $x_{0}$ is a solution of $(P)$ and $\Lambda_{1}$ is a solution of $(D)$, then there exists an element $x_{1} \in X$ such that $\left(x_{1}, \Lambda_{1}\right)$ is a feasible pair of $(D)$, for which either $F\left(x_{0}\right) \sim_{K} L\left(x_{1}, \Lambda_{1}\right)$ or they are not comparable w.r.t. $\prec_{K}$.

Proof. Let $y_{0}=\mu\left(F\left(x_{0}\right)\right)$. From the separation result provided in Theorem 6.2.5 in [15] and from Lemmas 3.9 and 3.10, there exists a pair $(\varphi, \psi) \in Y^{*} \times Z^{*} \backslash\{(0,0)\}$ such that $\forall(y, z) \in Q(X)$ and $\forall(k, c) \in(K, C)$ it holds

$$
\varphi(y)+\psi(z) \geq \varphi\left(y_{0}-k\right)+\psi(-c)
$$

Step 1. Let us show that that $\varphi \in K^{+}$and $\psi \in C^{+}$. From inequality (19), by letting $(y, z)=\left(y_{0}, 0\right) \in Q(X)$ and $c=0$, it holds $\varphi(-k) \leq 0, \forall k \in K$ which implies $\varphi \in K^{+}$. By letting $(y, z)=\left(y_{0}, 0\right) \in Q(X)$ and $k=0$, inequality (19) leads to $\psi(-c) \leq 0, \forall c \in C$ which implies $\psi \in C^{+}$.

Step 2. We now claim that $\varphi \neq 0$. Indeed, by contradiction, suppose $\varphi=0$. If $c=0$ is selected, then inequality (19) leads to $\psi(z) \geq 0$, which holds for all $z \in Z$ such that there exists $x \in X$ with $G(x) \prec_{C}\{z\}$. However, by SCQ, there exists an element $\hat{x} \in X$ such that $G(\hat{x}) \subseteq-\operatorname{int} C$. Let $\hat{z}=\mu(G(\hat{x}))$, since $\hat{z} \in G(\hat{x})$ then $\hat{z} \in-\operatorname{int} C$. Picking such a $\hat{z}$, the previous inequality reads as $\psi(\hat{z}) \geq 0$, which contradicts $\psi \in C^{+}$because of $\psi \neq 0$, which follows from $(\varphi, \psi) \neq(0,0)$ and $\varphi=0$.

Step 3. Let $z_{0}=\mu\left(G\left(x_{0}\right)\right)$. We claim that

$$
\psi\left(z_{0}\right)=0
$$

Indeed, by letting $(y, z)=\left(y_{0}, z_{0}\right) \in Q(X), k=0$ and $c=0$, inequality (19) leads to $\psi\left(z_{0}\right) \geq 0$. Since $x_{0}$ is a solution of $(P)$ then it is $G\left(x_{0}\right) \subseteq-C$, implying $z_{0}=$ $\mu\left(G\left(x_{0}\right)\right) \in-C$. Relation (20) follows from $\psi \in C^{+}$.

Step 4. Let the linear operator $\Lambda_{0} \in \mathcal{L}^{+}(Z, Y)$ be defined as

$$
\Lambda_{0}(z)=k_{0} \psi(z)
$$

where $k_{0} \in \operatorname{int} K$. We show that

$$
\mu\left(L\left(x_{0}, \Lambda_{0}\right)\right)=\mu\left(F\left(x_{0}\right)\right)=y_{0}
$$


Indeed, with no loss of generality, assume that $\varphi\left(k_{0}\right)=1$. Then, from Lemma 2.14, point $v$ ), it holds that $\mu\left(L\left(x_{0}, \Lambda_{0}\right)\right)=\mu\left(F\left(x_{0}\right)\right)+\Lambda_{0}\left(\mu\left(G\left(x_{0}\right)\right)\right)=y_{0}+\Lambda_{0}\left(z_{0}\right)$ where $z_{0}=\mu\left(G\left(x_{0}\right)\right)$. Therefore, from relations (20) and (21), equality (22) follows.

Step 5. Let us show that $\left(x_{0}, \Lambda_{0}\right)$ is a feasible pair for $(D)$, that is

$$
L\left(x_{0}, \Lambda_{0}\right) \in \Phi\left(\Lambda_{0}\right)
$$

where $\Phi\left(\Lambda_{0}\right)=\prec_{\mathrm{K}}-\min \left\{L\left(x, \Lambda_{0}\right): x \in X\right\}$. Indeed, by contradiction, suppose that there exists $x_{1} \in X$ such that

$$
F\left(x_{1}\right)+\left(\Lambda_{0} \circ G\right)\left(x_{1}\right) \prec_{K} F\left(x_{0}\right)+\left(\Lambda_{0} \circ G\right)\left(x_{0}\right) .
$$

Relation (24) and (22) imply that

$$
F\left(x_{1}\right)+\left(\Lambda_{0} \circ G\right)\left(x_{1}\right) \subseteq F\left(x_{0}\right)-\operatorname{int} K=y_{0}-\operatorname{int} K,
$$

i.e. $\forall y_{1} \in F\left(x_{1}\right)$ and $\forall z_{1} \in G\left(x_{1}\right)$ there exists $k^{1} \in \operatorname{int} K$ such that

$$
y_{1}+k^{0} \psi\left(z_{1}\right)=y_{0}-k^{1}
$$

Hence, applying $\varphi$ to (26), we get

$$
\varphi\left(y_{1}\right)+\psi\left(z_{1}\right)=\varphi\left(y_{0}\right)-\varphi\left(k^{1}\right)<\varphi\left(y_{0}\right)
$$

By choosing $\left(y_{1}, z_{1}\right)=\left(\mu\left(F\left(x_{1}\right)\right), \mu\left(G\left(x_{1}\right)\right)\right) \in Q(X)$, relation (27) contradicts the separation result (19) if the pair $(k, c)=(0,0)$ is selected. It follows $L\left(x_{0}, \Lambda_{0}\right) \in$ $\Phi\left(\Lambda_{0}\right)$, thus $\left(x_{0}, \Lambda_{0}\right)$ is a feasible pair of $(D)$.

Step 6. Finally, let us show that for every solution $\Lambda_{1}$ of $(D)$ there exists $x_{1} \in X$ such that $\left(x_{1}, \Lambda_{1}\right)$ is a feasible pair for $(D)$ and $L\left(x_{1}, \Lambda_{1}\right) \nprec_{K} F\left(x_{0}\right)$. Suppose, by contradiction, that for all $x_{1} \in X$ such that the pairs $\left(x_{1}, \Lambda_{1}\right)$ are feasible for $(D)$, it holds

$$
L\left(x_{1}, \Lambda_{1}\right) \prec_{K} F\left(x_{0}\right)
$$

According to (22) and Lemma 2.14 point ii), we can reformulate relation (28) as

$$
L\left(x_{1}, \Lambda_{1}\right) \subseteq F\left(x_{0}\right)-\operatorname{int} K=y_{0}-\operatorname{int} K=L\left(x_{0}, \Lambda_{0}\right)-\operatorname{int} K,
$$

which contradicts the fact that $\Lambda_{1}$ is a solution of $(D)$.

Step 7. From relation $L\left(x_{1}, \Lambda_{1}\right) \nprec_{K} F\left(x_{0}\right)$, together with Corollary 3.8, either $F\left(x_{0}\right) \sim_{K} L\left(x_{1}, \Lambda_{1}\right)$ or they are not comparable w.r.t. the order $\prec_{K}$ and the statement follows.

Corollary 3.13. Under assumptions of Theorem 3.12, for any solution $x_{0}$ of $(P)$ there exists a solution $\Lambda_{0}$ of $(D)$ such that

$$
F\left(x_{0}\right) \sim_{K} L\left(x_{0}, \Lambda_{0}\right)
$$

Proof. Let $x_{0}$ be a solution of $(P)$ and let $\Lambda_{0}$ be as in the proof of Theorem 3.12 . From equation (22) and Lemma 2.14 point $i i i)$ it holds

$$
F\left(x_{0}\right) \sim_{K} L\left(x_{0}, \Lambda_{0}\right)
$$

In the proof of Theorem 3.12, it has already been shown that the pair $\left(x_{0}, \Lambda_{0}\right)$ is feasible for $(D)$. Moreover, by Corollary 3.8 and relation (30), it follows that $\Lambda_{0}$ is a solution of $(D)$. 
Remark 3.14. Within the conduction of the proof of Theorem 3.12, we follow an approach used in [9] (see also [10]), where the authors exploit separation results to prove saddle-point theorems.

Even if our approach is similar, the fact that we consider a set minimization problem w.r.t. the upper order relation gives to our primal problem a peculiar structure. As a consequence, the sets that are separated in our theorem and the thesis we prove are different from those appearing in the cited works (see Theorems 3.5 and 4.2 in [9]). Strong duality, as formulated in Theorem 3.12, will be used in the sequel to state a result in the line of "dual best equals primal worst" (see [2]) within the context of uncertain vector programs.

We note further that Theorem 3.12 cannot be obtained as a direct adaptation of Theorem 3.5 in [9], named "strong duality" as well, with a change in the order relation among sets. In particular, we restrict our analysis to cone-convex objectives and constraints and formulate our strong duality result using the upper order relation among sets to compare, if possible, the images of the objective map $F$ and of the Lagrangian map $L$, of the dual problem.

Our formulation can be considered as a direct extension to the set optimization problem $(P)$ of the Lagrangian dual considered in [19]. We recall that in [19], even in the spacial case of a vector optimization primal, the set-valued dual is implicitly solved by the so called "vector criterion" introduced in [5].

Finally, we remark that Theorem 3.12 reduces, in the scalar case, to the classical strong Lagrangian duality theorem (see [1], among others), thus providing an explicit generalization of those results to set-valued problems under Assumption 3.6. Hence, we denote Theorem 3.12 as "strong duality".

Remark 3.15. We note that, under Assumption 3.6, we can associate to the setvalued primal problem $(P)$ an equivalent vector optimization problem

$$
\mathrm{WMin}_{K} \mu(F(x)) \text { s.t. } \mu(G(x)) \leq_{C} 0
$$

Indeed, by Lemma 2.14, point iv), an element $x_{0} \in X$ is a solution of $(P)$ if and only if it is a solution of $(p)$. Moreover, the $\prec_{K}$-convexity of $F$ and the $\prec_{C}$-convexity of $G$ imply that $(p)$ is a convex program. However, since the dual problem of a vector valued primal has an intrinsic set-valued nature (see e.g. [19], [14], where the solution of the set-valued dual problem is considered according to the so called vector criterion introduced by [5]), we choose a set optimization formulation for the primal that allows us to state the general weak duality result in Theorem 3.5 and to maintain a uniform set-valued treatment in Theorem 3.12.

\section{Robust vector optimization: pessimistic primal and opti- mistic dual}

Robust optimization is one of the basic methodologies to face optimization problems which depend on uncertain parameters. More precisely, robust optimization deals with the cases in which information on the uncertainties are limited to the knowledge of the sets where they range (see [4]). We aim at applying such a methodology to uncertain convex vector programs where both the objective as well as the constraint depend on uncertain parameters to obtain a result in the sense of "primal worst equals dual best", provided by [2] for the scalar case. To this purpose we extend the concept of minmax robustness, introduced by [21] and widely developed in [3] for scalar cases, to vector uncertain optimization problems as in [11] (see also [6] and [18] for the special case in which $Y=\mathbb{R}^{m}$ ordered by the cone $K=\mathbb{R}_{+}^{m}$ and 
$Z=\mathbb{R}^{k}$ ordered by the cone $C=\mathbb{R}_{+}^{k}$ ). For a recent survey on robust multiobjective optimization, see also [22] and [12].

Let us consider the family of parametric vector optimization problems:

$$
\mathrm{WMin}_{x} f(x, v) \quad \text { subject to } g(x, u) \leq_{C} 0 \quad(U-P)
$$

The robust approach hedges against worst cases and only the values of the objective in the worst possible realizations of parameters are of interest. In order to formulate the robust counterpart of $(U-P)$ as a deterministic set-valued program, let us consider the set of objective values at the decision variable $x$ :

$$
F(x):=\{f(x, v): v \in V\} \subseteq Y
$$

Before proceeding, it should be noted that also the compliance with the constraints is conditioned upon the parameters' realizations. In order to introduce the concept of robust feasible elements we refer to [4], where the authors note that for real-world optimization problems the constraints must remain feasible for all the realizations of the data. Then, robust feasible elements are those elements $x \in X$ for which the condition $g(x, u) \leq_{C} 0$ is satisfied for all $u \in U$, worst cases included. In this view, consider the set of constraint values at $x$

$$
G(x):=\{g(x, u): u \in U\} \subseteq Z
$$

The correspondence (32) defines the set-valued map $G: X \rightrightarrows Z$ that is used to express the feasible region of the robust counterpart of $(U-P)$. Indeed, an element $x \in X$ is robust feasible provided that $G(x) \subseteq-C$ or, equivalently, $G(x) \prec_{C}\{0\}$.

The following set-valued optimization problem can be interpreted as the robust counterpart of the uncertain problems $(U-P)$ since it takes into account the worst case scenarios:

$$
\prec_{\mathrm{K}}-\min F(x) \quad \text { subject to } \quad G(x) \prec_{C}\{0\} \quad(R C-P)
$$

Remark 4.1. Solutions of $(R C-P)$ can be considered as robust weakly efficient solutions, defined in [6], in presence of a parametric constraint, for the special case in which $Y=\mathbb{R}^{m}$ ordered by the cone $K=\mathbb{R}_{+}^{m}$ and $Z=\mathbb{R}^{k}$ ordered by the cone $C=\mathbb{R}_{+}^{k}$.

Similarly to what was done within the formulation of the robust counterpart of the uncertain primal problems $(U-P)$, we aim at associating to their uncertain dual problems $(U-D)$ an optimistic counterpart, consisting in a deterministic set-valued program where the parameters' realizations are assumed to be the most favorable ones. The goal is to establish a relationship among the image sets of the solutions of the robust primal and of the set of solutions of optimistic dual. Let $\Lambda \in \mathcal{L}^{+}(Z, Y)$ be a monotone linear operator from $Z$ to $Y$ and let us consider the uncertain Lagrangian function associated to problem $(U-P)$ :

$$
l(x, \Lambda, v, u)=f(x, v)+\Lambda(g(x, u))
$$

In terms of the uncertain Lagrangian, the family of parametric set-valued dual problems $(U-D)$ can be formulated as an uncertain set-valued maximization with respect to $\Lambda \in \mathcal{L}(Z, Y)$ of the following parametric objective

$$
\operatorname{WMin}_{K}\{l(x, \Lambda, v, u): x \in X\}
$$


We remark that, for a given pair $(v, u),(34)$ is a vector minimization problem where the optimal values are, in general, sets and not singletons; hence the maxmin problem $(U-D)$ is intrinsically set-valued. We define the solution concept of $(U-D)$, adapting to our set optimization setting the approach considered in [19] with reference to the so called "vector criterion", introduced in [5]: given a pair $(v, u), \Lambda_{1}$ solves $(U-D)$ if there exists $x_{1} \in X$ such that $l\left(x_{1}, \Lambda_{1}, v, u\right) \in \operatorname{Wmin}_{K}\left\{l\left(x, \Lambda_{1}, v, u\right), x \in X\right\}$ and, for all pairs $\left(x^{\prime}, \Lambda^{\prime}\right)$ such that $l\left(x^{\prime}, \Lambda^{\prime}, v, u\right) \in \operatorname{Wmin}_{K}\left\{l\left(x, \Lambda^{\prime}, v, u\right): x \in X\right\}$ it holds

$$
l\left(x_{1}, \Lambda_{1}, v, u\right) \nless_{K} l\left(x^{\prime}, \Lambda^{\prime}, v, u\right)
$$

Now, we define a notion of optimistic counterpart of $(U-D)$ by means of the quasiorder relation $\prec_{K}$ that allows us to consider only best scenarios in the bilevel parametric dual problem $(U-D)$. To this purpose, let us consider the set of Lagrangian values at $(x, \Lambda)$ :

$$
L(x, \Lambda)=\{l(x, \Lambda, v, u):(v, u) \in V \times U\}
$$

We say that $\Lambda_{1} \in \mathcal{L}^{+}(Z, Y)$ is an optimistic solution of $(U-D)$ if there exists $x_{1} \in X$ such that

$$
L\left(x_{1}, \Lambda_{1}\right) \in\left\{\prec_{K}-\min \left\{L\left(x, \Lambda_{1}\right), x \in X\right\}\right\}
$$

and, for all pairs $\left(x^{\prime}, \Lambda^{\prime}\right)$ such that

$$
L\left(x^{\prime}, \Lambda^{\prime}\right) \in\left\{\prec_{K}-\min \left\{L\left(x, \Lambda^{\prime}\right): x \in X\right\}\right\}
$$

it holds

$$
L\left(x_{1}, \Lambda_{1}\right) \nprec_{K} L\left(x^{\prime}, \Lambda^{\prime}\right)
$$

Hence, by introducing the collections of sets

$$
\Phi(\Lambda)=\prec_{\mathrm{K}}-\min \{L(x, \Lambda): x \in X\}, \text { for all } \Lambda \in \mathcal{L}^{+}(Z, Y)
$$

we reformulate the optimistic counterpart $(O C-D)$ of the parametric dual $(U-D)$ as

$$
\prec_{\mathrm{K}}-\operatorname{MAX} \Phi(\Lambda) \quad \text { subject to } \Lambda \in \mathcal{L}^{+}(Z, Y) \quad(O C-D)
$$

where the " $\prec_{K}-M A X$ " operator is defined in Section 3 .

Remark 4.2. The interpretation of $\Lambda_{1}$ as an optimistic solution of the uncertain dual problem $(U-D)$ is more explicit under the special case in which Assumption 3.6 holds. Indeed, in this event, at each $(x, \Lambda) \in X \times \mathcal{L}^{+}(Z, Y)$ the nadir $\mu(L(x, \Lambda))$ represents the best scenario at $(x, \Lambda)$, since

$$
\mu(L(x, \Lambda))=\operatorname{Max}_{K}\{l(x, \Lambda, v, u):(v, u) \in V \times U\}
$$

Then, according with the previous definition of optimistic solution, it follows that $\Lambda_{1}$ solves $(O C-D)$ if there exists $x_{1} \in X$ such that

$$
\mu\left(L\left(x_{1}, \Lambda_{1}\right) \in \operatorname{Wmin}_{K}\left\{\mu\left(L\left(x, \Lambda_{1}\right)\right): x \in X\right\}\right.
$$

and, for all pairs $\left(x^{\prime}, \Lambda^{\prime}\right)$ such that

$$
\mu\left(L\left(x^{\prime}, \Lambda^{\prime}\right)\right) \in \mathrm{Wmin}_{K}\left\{\mu\left(L\left(x, \Lambda^{\prime}\right)\right): x \in X\right\}
$$

it holds

$$
\mu\left(L\left(x_{1}, \Lambda_{1}\right)\right) \nless_{K} \mu\left(L\left(x^{\prime}, \Lambda^{\prime}\right)\right)
$$

Hence, under Assumption 3.6, we can reformulate the duality result as a maxmin problem on best scenarios.

Remark 4.3. We remark that problem $(O C-D)$ does not reduce, in the scalar case, to the optimistic counterpart (OD-P) considered in [2], but only to the so called dual robust problem (DR-P) without any additional concavity assumptions. 


\section{Primal worst and dual best in vector optimization}

In this section we aim at highlighting the relationship among the image solutions of the robust counterpart of an uncertain vector problem and the image solutions of the optimistic counterpart of the related uncertain dual. This is along the line marked by [2] where the authors show that, for a scalar uncertain convex program, optimizing under the worst case scenario in the primal is the same as optimizing under the best case scenario in the dual ("primal worst equals dual best"). We note that, within the present context, the robust counterpart and the optimistic dual have an intrinsic set-valued nature. Following a set optimization approach, we compare sets under a partial quasiorder. Hence, we may find pairs of non comparable sets and nontrivial equivalence classes.

Relations between the image sets of the robust counterpart and the image sets of the optimistic dual are obtained through the weak duality (see Theorem 3.5), which leads to the following result.

Theorem 5.1. Let $x_{0}$ be a feasible element of $(R C-P)$ and $\left(x_{1}, \Lambda_{1}\right)$ be a feasible pair of $(O C-D)$. If $F\left(x_{0}\right) \prec_{K} L\left(x_{1}, \Lambda_{1}\right)$, then $F\left(x_{0}\right) \sim_{K} L\left(x_{1}, \Lambda_{1}\right)$

Proof. By noting that the optimistic counterpart $(O C-D)$ is the set-valued dual of the robust counterpart $(R C-P)$, as defined in Section 3, the thesis follows from Theorem 3.5.

Further characterizations of relations between the image sets of the robust counterpart and the image sets of the optimistic dual are obtained through the strong duality result (see Theorem 3.12), which is proven for set-valued programs where both the image sets of the objective and of the constraint contain their nadir points for any value of the decision variable (see Assumption 3.6). With reference to the uncertain problems $(U-P)$, we note that Assumption 3.6 is equivalently stated as follows.

Assumption 5.2. A pair of uncertainties $(\bar{v}(x), \bar{u}(x)) \in V \times U$ at any given value $x$ of the decision variable such that

$$
f(x, v) \leq_{K} f(x, \bar{v}(x)) \text { and } g(x, u) \leq_{C} g(x, \bar{u}(x)) \text { for any }(v, u) \in V \times U
$$

exists.

Relations (37) imply $f(x, \bar{v}(x))=\mu(F(x))$ and $g(x, \bar{u}(x))=\mu(G(x))$ and the pair $(\bar{v}(x), \bar{u}(x))$ represents the unique worst case scenario at $x$. Moreover, we will see in Section 6 that such an assumption, implicitly considered e.g. in [8], is not unrealistic in many applications.

Remark 5.3. The assumption that the worst case scenario at $x$, corresponding to the nadir point $\mu(F(x)=f(x, v(\bar{x}))$, can be achieved is not realistic in many applications. This may occur when the uncertainty on the objective vector-valued function cannot be described componentwise, for instance because there is negative correlation among uncertainties on distinct components of the objective function. If one considers a typical portfolio selection model where uncertainties on expected returns and volatility are deeply correlated, the assumption that worst case scenarios are achieved is usually not satisfied. For instance, in the robust multiobjective portfolio optimization model introduced in [7], it can be clearly seen (in Fig.2, p. 431) that nadir points of the uncertainty sets are not achieved, hence the robustification procedure proposed there may be unduly restrictive. 
Theorem 5.4. Consider the vector problem $(U-P)$ under Assumption 5.2. Assume that the decision space $X$ is convex, the set-valued maps $F(x)=\{f(x, v): v \in V\}$ and $G(x)=\{g(x, u): u \in U\}$ are respectively $\prec_{K}$-convex and $\prec_{C^{-} \text {-convex on } X \text { and }}$ there exists $\hat{x}$ such that $g(\hat{x}, u)<_{C} 0$ for all $u \in U$ (SCQ). Then, if $x_{0}$ solves $(R C-P)$ and $\Lambda_{1}$ solves $(O C-D)$, there exists an element $x_{1}$ such that the pair $\left(x_{1}, \Lambda_{1}\right)$ is feasible for $(O C-D)$ and either

$$
F\left(x_{0}\right) \sim_{K} L\left(x_{1}, \Lambda_{1}\right)
$$

or $F\left(x_{0}\right)$ and $L\left(x_{1}, \Lambda_{1}\right)$ are not comparable w.r.t. $\prec_{K}$.

Proof. Relation (38) follows from the strong duality Theorem 3.12.

The following formulation follows from Corollary 3.13.

Corollary 5.5. Under all the assumptions of Theorem 5.4, if $x_{0}$ solves $(R C-P)$ there exists $\Lambda_{0} \in \mathcal{L}^{+}(Z, Y)$ such that $\left(x_{0}, \Lambda_{0}\right)$ is feasible for $(D), \Lambda_{0}$ solves $(O C-D)$ and

$$
F\left(x_{0}\right) \sim_{K} L\left(x_{0}, \Lambda_{0}\right)
$$

Relations (38) and (39) imply that operating under the worst possible data in the primal is neither better nor worse than operating under the best possible data in the dual. Then the solutions of the two set optimization problems $(R C-P)$ and $(O C-D)$ correspond to equivalent or not comparable image sets w.r.t. $\prec_{K}$. Moreover, corollary 5.5, ensures that equivalence can be reached by choosing the solution of the optimistic dual in the form (21).

Remark 5.6. Under Assumption 5.2, the existence of a worst case scenario at each decision variable allows us to formulate the robust counterpart $(R C-P)$ as a vector valued problem (as in [8], for the special case considered there)

$$
\mathrm{WMin}_{K} \mu(F(x)) \text { s.t. } \mu(G(x)) \leq_{C} 0 \quad(r c-p)
$$

However we maintain a homogeneous set-valued approach consistent with Theorem 5.1, regardless Assumption 5.2. More generally, we conduct the treatment of the robust counterpart coherently with the existing literature (see e.g. [6], [11]). We remark also that when worst case scenarios cannot be achieved, notions such as objective-wise worst case considered in [6] and introduced in [18] may lead to a robust solution concept that does not necessarily correspond to the one considered here. Hence, without Assumption 5.2, the vector optimization formulation $(r c-p)$ is not coherent with our approach. Finally, we observe that problem $(O C-D)$ has an intrinsic set-valued nature (see to this purpose Remark 3.15).

We highlight that the convexity assumptions in our results are different from the ones considered in [2]. The following lemma establishes a sufficient condition for the

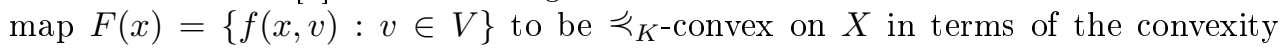
properties of the vector function $f$.

Lemma 5.7. Let $X$ be a convex set and let the vector function $f: X \times V \longrightarrow Y$ be convex on $X$. Then the set-valued map $F: X \rightrightarrows Y$ defined at any $x \in X$ as $F(x)=\{f(x, v): v \in V\}$ is $\prec_{K}$-convex.

Proof. Because of the convexity of $f$ w.r.t. its first variable, it results that

$$
f\left(\alpha x_{1}+(1-\alpha) x_{2}, v\right) \in \alpha f\left(x_{1}, v\right)+(1-\alpha) f\left(x_{1}, v\right)-K
$$


The previous relation holds true for any $v \in V$, so one can consider the union over the set $V$, which leads to

$$
F\left(\alpha x_{1}+(1-\alpha) x_{2}\right) \subseteq \alpha F\left(x_{1}\right)+(1-\alpha) F\left(x_{1}\right)-K
$$

Then, according to Definition (2.10), $F$ is $\prec_{K}$-convex.

Hence the convexity properties of $(R C-P)$ can be inherited from those of $(U-P)$. Indeed, if the objective $f$ and the constraint $g$ of an uncertain vector problem are convex w.r.t. the decision variable $x$, then the objective $F$ and the constraint $G$ of the robust counterpart are $\prec_{K}$-convex and $\prec_{C}$-convex respectively. Conversely, if $F$ and $G$ are $\prec_{K}$-convex and $\prec_{C}$-convex respectively, the objective and the constraint of the uncertain problems need not to be convex w.r.t. the decision variable. This is shown in the following example.

Example 5.8. Consider the uncertain problem

$$
\mathrm{Wmin}_{\mathbb{R}_{+}}-v x^{2} \text { subject to }-x \leq_{\mathbb{R}_{+}} 0 \quad(U-P(\text { Ex. 5.8) })
$$

where $x \in \mathbb{R}, v \in[0,1]$ and $\mathbb{R}_{+}=\{x \in \mathbb{R}: 0 \leq x\}$ is the ordering cone. Note that the objective $f(x, v)=-v x^{2}$ is not convex w.r.t. $x$ but the set-valued map $F(x)=\left\{-v x^{2}: v \in[0,1]\right\}$ is $\prec_{\mathbb{R}_{+}}$-convex.

Note that also $G$ is $\prec_{\mathbb{R}_{+}}$-convex, being $G(x)=\{-x\}$, and for any $\hat{x} \in\{x \in \mathbb{R}$ : $x<0\}$ it is $G(\hat{x}) \prec_{\mathbb{R}_{+}}\{0\}$ (SCQ). In addition, $\mu(F(x))=\{0\}$ and $\mu(G(x))=\{-x\}$. Hence all the hypotheses of Theorem 5.4 are fulfilled.

Moreover, for a complete comparison with the convexity assumptions in [2], we show with an example that, if $F$ and $G$ are $\prec_{K^{-}}$-convex and $\prec_{C}$-convex respectively, the objective and the constraint of $(U-P)$ are not necessarily concave w.r.t. the uncertain parameters.

Example 5.9. Consider the uncertain problem

$$
\mathrm{WMin}_{\mathbb{R}_{+}} v^{2} x \text { subject to }-x \leq_{\mathbb{R}_{+}} 0 \quad(U-P(\text { Ex. } 5.9))
$$

where $x \in \mathbb{R}, v \in[0,1]$ and $\mathbb{R}_{+}=\{x \in \mathbb{R}: 0 \leq x\}$ is the ordering cone. Note that $f(x, v)=v^{2} x$ is not concave w.r.t. $v$ but the set-valued map $F(x)=\left\{v^{2} x: v \in[0,1]\right\}$ is $\prec_{\mathbb{R}_{+}}$-convex.

Note that also $G$ is $\prec_{\mathbb{R}_{+}}$-convex, being $G(x)=\{-x\}$, and for any $\hat{x} \in\{x \in \mathbb{R}$ : $x<0\}$ it is $G(\hat{x}) \prec_{\mathbb{R}_{+}}\{0\}$ (SCQ). In addition, $\mu(F(x))=\{x\}$ and $\mu(G(x))=\{-x\}$. Hence all the hypotheses of Theorem 5.4 are fulfilled.

\section{Application to componentwise uncertain multiobjective pro- gramming}

In many practical instances, the existence of pessimistic nadir points for the sets of values of the objective and of the constraint at any given value of the decision variable, occurs. For example, in multiobjective optimization, we can consider the case of componentwise uncertainties where the uncertainties in the components of the objective and of the constraint vector functions are independent of each other. We define componentwise uncertain multiobjective problems by extending the definition of objective wise uncertain problems provided in [6] to the case where also the feasible region is uncertain and expressed by means of inequalities. 
Definition 6.1. A multiobjective problem $(U-P)$ where $(v, u) \in V \times U \subset \mathbb{R}^{n} \times \mathbb{R}^{m}$, is componentwise uncertain if the uncertainties of each component of the objective $f: X \times V \longrightarrow \mathbb{R}^{n}$ and of the constraint $g: X \times U \longrightarrow \mathbb{R}^{m}$ are independent of each other, namely if $V=V_{1} \times V_{2} \ldots V_{n}$ with $V_{i} \subset \mathbb{R}^{n_{i}}$ and $\sum_{i} n_{i}=n, U=U_{1} \times U_{2} \ldots U_{m}$ with $U_{j} \subset \mathbb{R}^{m_{j}}$ and $\sum_{j} m_{j}=m$ and

$$
f(x, v)=\left(\begin{array}{c}
f_{1}\left(x, v_{1}\right) \\
f_{2}\left(x, v_{2}\right) \\
\vdots \\
f_{n}\left(x, v_{n}\right)
\end{array}\right), g(x, u)=\left(\begin{array}{c}
g_{1}\left(x, u_{1}\right) \\
g_{2}\left(x, u_{2}\right) \\
\vdots \\
g_{m}\left(x, u_{m}\right)
\end{array}\right)
$$

Under suitable assumptions, the following lemma shows that for multiobjective problems with componentwise uncertainties a worst case in the parameter space exists at any given $x$.

Lemma 6.2. See [6], Lemma 5.2. Let $(P(u, v))$ be a componentwise uncertain multiobjective problem. If $\max _{v \in V} f_{i}(x, v)$ and $\max _{u \in U} g_{j}(x, u)$ exist for any $x \in X$, $i=1, \ldots, n$ and $j=1, \ldots, m$, then

$$
\bar{v}(x):=\left(\begin{array}{c}
\arg \max _{v_{1} \in V} f_{1}\left(x, v_{1}\right) \\
\arg \max _{v_{2} \in V} f_{2}\left(x, v_{2}\right) \\
\vdots \\
\arg \max _{v_{n} \in V} f_{n}\left(x, v_{n}\right)
\end{array}\right) \in V, \quad \bar{u}(x)=\left(\begin{array}{c}
\arg \max _{u_{1} \in U} g_{1}\left(x, u_{1}\right) \\
\arg \max _{u_{2} \in U} g_{2}\left(x, u_{2}\right) \\
\vdots \\
\arg \max _{u_{m} \in U} g_{m}\left(x, u_{m}\right)
\end{array}\right) \in U
$$

Remark 6.3. It follows immediately that $f(x, \bar{v}(x))=\mu(F(x))$ and $g(x, \bar{u}(x))=$ $\mu(G(x))$, where $F(x)=\{f(x, v): v \in V\}$ and $G(x)=\{g(x, u): u \in U\}$, since

$$
f(x, v) \leq_{\mathbb{R}_{+}^{n}} f(x, \bar{v}(x)), \forall v \in V \text { and } g(x, u) \leq_{\mathbb{R}_{+}^{m}} g(x, \bar{u}(x)), \forall u \in U
$$

\subsection{Multiobjective supplier selection model}

An important class of componentwise uncertain multiobjective programs are multiobjective linear programs (MOLP) with interval coefficients (see [20] and the references therein for an overview). Such uncertain programs arise when the interval programming approach is applied to MOLPs. For the sake of completeness, we recall that the interval programming approach is devoted to provide decision support models for real-world situations, where the coefficients involved in optimization programs are not exactly known. Interval programming tackle indeterminacy by means of confidence intervals for the uncertain coefficients, just requiring the indication of lower and upper bounds and with no need to specify empirical or postulated distributions.

We show here that our results can be applied to the multiobjective uncertain problems studied in [13]. The authors consider a single item supplier selection problem (SSP), modeled as a MOLP, where the decision maker (company) considers three criteria, that are purchasing cost, rejects and lead-times. Let us denote by $n$ the number of suppliers and let $x_{i} \in \mathbb{R}$ be the amount of items ordered from supplier $i$. Moreover, denote by

$u_{i}$ the capacity of supplier $i$;

$c_{i}$ the unit purchasing price from supplier $i$;

$q_{i}$ the expected defect rate of supplier $i$;

$p_{i}$ the percentage of items delivered late by supplier $i$. 
The SSP formulated in [13] can be adapted in our framework as

$$
\mathrm{Wmin}_{\mathrm{R}_{+}^{3}} f(x) \text { subject to } g(x) \leq_{\mathbb{R}_{+}^{2 n+1}} 0
$$

where the parametric objective $f: \mathbb{R}^{n} \longrightarrow \mathbb{R}^{3}$ and the parametric constraint $g$ : $\mathbb{R}^{n} \longrightarrow \mathbb{R}^{2 n+1}$ are given by

$$
f(x)=\left[\begin{array}{c}
\sum_{i=1}^{n} c_{i} x_{i} \\
\sum_{i=1}^{n} q_{i} x_{i} \\
\sum_{i=1}^{n} p_{i} x_{i}
\end{array}\right] \text { and } g(x)=\left[\begin{array}{c}
d-\sum_{i=1}^{n} x_{i} \\
x_{1}-u_{1} \\
x_{2}-u_{2} \\
\vdots \\
x_{n}-u_{n} \\
-x_{1} \\
-x_{2} \\
\vdots \\
-x_{n}
\end{array}\right]
$$

where $d$ is the demand and $x \in \mathbb{R}^{n}$. Let us now account for the uncertainties within the suppliers capacities and services. By applying the interval programming approach, let us assume that the demand $d$ and the parameters $u_{i}, c_{i}, q_{i}, p_{i}$, for all $i=1, \ldots, n$, are uncertain and their lower and the upper bounds are given. The uncertainty sets $V$ and $U$, which are related to the parameters entering into the objective and into the constraint respectively, are defined as

$$
V=\prod_{i=1}^{n}\left[\underline{c}_{i}, \bar{c}_{i}\right] \times \prod_{i=1}^{n}\left[\underline{q}_{i}, \bar{q}_{i}\right] \times \prod_{i=1}^{n}\left[\underline{p}_{i}, \bar{p}_{i}\right] \text { and } U=[\underline{d}, \bar{d}] \times \prod_{i=1}^{n}\left[\underline{u}_{i}, \bar{u}_{i}\right]
$$

where under-signed and over-signed values denote lower and upper bounds of uncertain parameters. Under this assumption, problem $(S S P)$ becomes a MOLP with interval coefficients. We reformulate it as componentwise uncertain multiobjective problem, which reads as

$$
\mathrm{WMin}_{\mathbb{R}_{+}^{3}} f(x, v) \text { subject to } g(x, u) \leq_{\mathbb{R}_{+}^{2 n+1}} 0 \quad(U-S S P)
$$

where $(v, u) \in V \times U$ are the uncertainties.

The robust counterpart of $(U-S S P)$ is the following set-valued program

$$
\prec_{\mathbb{R}_{+}^{3}}-\min F(x) \text { subject to } G(x) \prec_{\mathbb{R}_{+}^{2 n+1}}\{0\} \quad(R C-S S P)
$$

where, for all $x \in \mathbb{R}^{n}$, it is $F(x)=\{f(x, v): v \in V\}, G(x)=\{g(x, u): u \in U\}$ and

$$
\mu(F(x))=\left[\begin{array}{c}
\sum_{i=1}^{n} \bar{c}_{i} x_{i} \\
\sum_{i=1}^{n} \bar{q}_{i} x_{i} \\
\sum_{i=1}^{n} \bar{p}_{i} x_{i}
\end{array}\right] \text { and } \mu(G(x))=\left[\begin{array}{c}
\bar{d}-\sum_{i=1}^{n} x_{i} \\
x_{1}-\underline{u}_{1} \\
x_{2}-\underline{u}_{2} \\
\vdots \\
x_{n}-\underline{u}_{n} \\
-x_{1} \\
-x_{2} \\
\vdots \\
-x_{n}
\end{array}\right]
$$


The uncertain dual problems of $(U-S S P)$ are

$$
\prec_{\mathbb{R}_{+}^{3}}-\max \phi(\Lambda, v, u) \text { subject to } \Lambda \in \mathcal{L}^{+}(Z, Y) \quad(D-S S P)
$$

where $\phi(\Lambda, v, u)=\mathrm{WMin}_{\mathbb{R}_{+}^{3}}\{l(x, \Lambda, v, u): x \in X\}, \Lambda$ is a $3 \times(2 n+1)$ matrix

$$
\Lambda=\left[\begin{array}{cccc}
\lambda_{1,1} & \lambda_{1,2} & \ldots & \lambda_{1,2 n+1} \\
\lambda_{2,1} & \lambda_{2,2} & \ldots & \lambda_{2,2 n+1} \\
\lambda_{3,1} & \lambda_{3,2} & \ldots & \lambda_{3,2 n+1}
\end{array}\right]
$$

with $\lambda_{i, j} \geq 0$ and the uncertain Lagrangian is

$$
\begin{aligned}
l(x, \Lambda, v, u)= & f(x, v)+\Lambda(g(x, u)) \\
= & {\left[\begin{array}{l}
\sum_{i=1}^{n} c_{i} x_{i}+\lambda_{1,1}\left(d-\sum_{i=1}^{n} x_{i}\right)+\sum_{i=1}^{n} \lambda_{1, i+1}\left(x_{i}-u_{i}\right)-\sum_{i=1}^{n} \lambda_{1, i+n+1} x_{i} \\
\sum_{i=1}^{n} q_{i} x_{i}+\lambda_{2,1}\left(d-\sum_{i=1}^{n} x_{i}\right)+\sum_{i=1}^{n} \lambda_{2, i+1}\left(x_{i}-u_{i}\right)-\sum_{i=1}^{n} \lambda_{2, i+n+1} x_{i} \\
\sum_{i=1}^{n} p_{i} x_{i}+\lambda_{3,1}\left(d-\sum_{i=1}^{n} x_{i}\right)+\sum_{i=1}^{n} \lambda_{3, i+1}\left(x_{i}-u_{i}\right)-\sum_{i=1}^{n} \lambda_{3, i+n+1} x_{i}
\end{array}\right] }
\end{aligned}
$$

The optimistic counterpart of $(D-S S P)$ is the following set-valued program

$$
\prec_{\mathbb{R}_{+}^{3}}-\operatorname{MAX} \Phi(\Lambda) \text { subject to } \Lambda \in \mathcal{L}^{+}(Z, Y) \quad(O C-S S P)
$$

where $\Phi(\Lambda)=\prec_{\mathbb{R}_{+}^{3}}-\min \left\{L(x, \Lambda): x \in \mathbb{R}^{n}\right\}$ and, at any $(x, \Lambda)$, the set-valued Lagrangian is $L(x, \Lambda)=\{f(x, v)+\Lambda(g(x, u)):(v, u) \in V \times U\}$ with $\mu(L(x, \Lambda))=$ $\mu(F(x))+\Lambda(\mu(G(x)))$.

\section{Conclusions}

We formulate the robust counterpart of an uncertain vector optimization problem and the optimistic counterpart of its uncertain dual. The resulting deterministic programs have an intrinsic set-valued nature. Following a set optimization approach, we compare the sets in the image space of the objective maps by means of a partial quasiorder with the aim of providing a result in the sense of "primal worst equal dual best" by [2]. For our result to be valid we require the cone-convexity of the set-valued objective and constraint involved in the robust counterpart. Moreover, we limit our attention to those uncertain primal optimization problems where, at each given value of the decision variable, pessimistic nadir points exist. In future works we aim at weakening such an assumption by extending the strong duality Theorem in Section 3 to generic cone-convex set-valued maps.

Acknowledgement 7.1. The third author is partially supported by Ministerio de Economìa y Competitividad (Spain), Project MTM2015-68103-P (MINECO/FEDER).

We thank an anonymous referee for his/her useful comments that contribute to improve this work.

\section{References}

[1] Mokhtar S. Bazaraa, Hanif D. Sherali, and Chitharanjan M Shetty. Nonlinear programming: theory and algorithms. John Wiley \& Sons, 2013.

[2] Amir Beck and Aharon Ben-Tal. Duality in robust optimization: primal worst equals dual best. Operations Research Letters, 37(1):1-6, 2009.

[3] Aharon Ben-Tal, Laurent El Ghaoui, and Arkadi Nemirovski. Robust optimization. Princeton University Press, 2009.

[4] Aharon Ben-Tal and Arkadi Nemirovski. Robust optimization-methodology and applications. Mathematical Programming, 92(3):453-480, 2002. 
[5] H.W. Corley. Existence and lagrangian duality for maximizations of set-valued functions. Journal of Optimization Theory and Applications, 54(3):489-501, 1987.

[6] Matthias Ehrgott, Jonas Ide, and Anita Schöbel. Minmax robustness for multiobjective optimization problems. European Journal of Operational Research, 239(1):17-31, 2014.

[7] Jörg Fliege and Ralf Werner. Robust multiobjective optimization \& applications in portfolio optimization. European Journal of Operational Research, 234(2):422-433, 2014.

[8] Miguel A Goberna, Vaithilingam Jeyakumar, Guoyin Li, and José Vicente-Pérez. Robust solutions to multi-objective linear programs with uncertain data. European Journal of Operational Research, 242(3):730-743, 2015.

[9] Elvira Hernández and Luis Rodríguez-Marín. Duality in set optimization with set-valued maps. Pac. J. Optim., 3(2):245-255, 2007.

[10] Elvira Hernández and Luis Rodríguez-Marín. Lagrangian duality in set-valued optimization. Journal of Optimization Theory and Applications, 134(1):119-134, 2007.

[11] Jonas Ide, Elisabeth Köbis, Daishi Kuroiwa, Anita Schöbel, and Christiane Tammer. The relationship between multi-objective robustness concepts and setvalued optimization. Fixed Point Theory and Applications, 2014(1):83, 2014.

[12] Jonas Ide and Anita Schöbel. Robustness for uncertain multi-objective optimization: a survey and analysis of different concepts. OR spectrum, 38(1):235, 2016.

[13] Omid Jadidi, Sergio Cavalieri, and Saeed Zolfaghari. An improved multi-choice goal programming approach for supplier selection problems. Applied Mathematical Modelling, 39(14):4213-4222, 2015.

[14] Johannes Jahn. Vector optimization. Springer, 2009.

[15] Akhtar A. Khan, Christiane Tammer, and Constantin Zalinescu. Set-valued optimization. Springer, 2015.

[16] Daishi Kuroiwa. On set-valued optimization. Nonlinear Analysis: Theory, Methods \&S Applications, 47(2):1395-1400, 2001.

[17] Daishi Kuroiwa. Existence theorems of set optimization with set-valued maps. Journal of Information and Optimization sciences, 24(1):73-84, 2003.

[18] Daishi Kuroiwa and Gue Myung Lee. On robust multiobjective optimization. Vietnam J. Math, 40(2-3):305-317, 2012.

[19] Dinh The Luc. Theory of vector optimization. lectures notes in economics and mathematical systems. 1989.

[20] Carla Oliveira and Carlos Henggeler Antunes. Multiple objective linear programming models with interval coefficients-an illustrated overview. European journal of operational Research, 181(3):1434-1463, 2007.

[21] Allen L. Soyster. Convex programming with set-inclusive constraints and applications to inexact linear programming. Operations research, 21(5):1154-1157, 1973.

[22] Margaret M Wiecek and Garrett M Dranichak. Robust multiobjective optimization for decision making under uncertainty and conflict. In Optimization challenges in complex, networked and risky systems, pages 84-114. INFORMS, 2016. 